\title{
Shedding light on auxin action
}

DOI:

10.1038/nrm2167

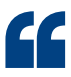

The unusual mechanism by which auxin activates SCF ${ }^{\mathrm{TIR} 1}$ might have significance beyond plant biology...
Auxin is a crucial plant hormone that controls many aspects of plant growth and development. Although auxin has been studied for over a century, its cellular receptor has only recently been discovered. Auxin binds to the F-box protein transport inhibitor response-1 (TIR1), which is part of the ubiquitin ligase SCF $^{\text {TIR1 }}$ complex, to regulate gene transcription through the ubiquitin-dependent degradation of the Aux/IAA family of transcriptional repressors. Zheng and colleagues have determined structures of TIR1 in complex with the SCF ${ }^{\mathrm{TIR} 1}$ adaptor protein ASK1 to reveal how auxin activates TIR1.

The structure of the TIR1-ASK1 complex is mushroom shaped, with much of TIR1 contributing to the mushroom cap. The auxin-binding pocket is formed by a Leu-rich repeat
(LRR) domain of TIR1 in the cap surface. Most strikingly, structures of the TIR-ASK1 complex, both with and without auxin bound, indicate that auxin does not induce large conformational changes in the structure of TIR-ASK1 upon binding. Therefore, auxin is not an allosteric regulator of $\mathrm{SCF}^{\mathrm{TIR} 1}$.

At the base of the auxin-binding pocket lies an inositol hexakisphosphate $\left(\mathrm{InsP}_{6}\right)$ molecule. This could be a structural cofactor that is required for the detection of auxin by TIR1. And considering that InsP $_{6}$ could not be removed under various conditions, it might be constitutively present. Auxin, and a number of other auxin-mimetic molecules, bind above InsP $\mathrm{P}_{6}$.

The structures of TIR1-ASK in complex with different auxin compounds revealed that the binding site

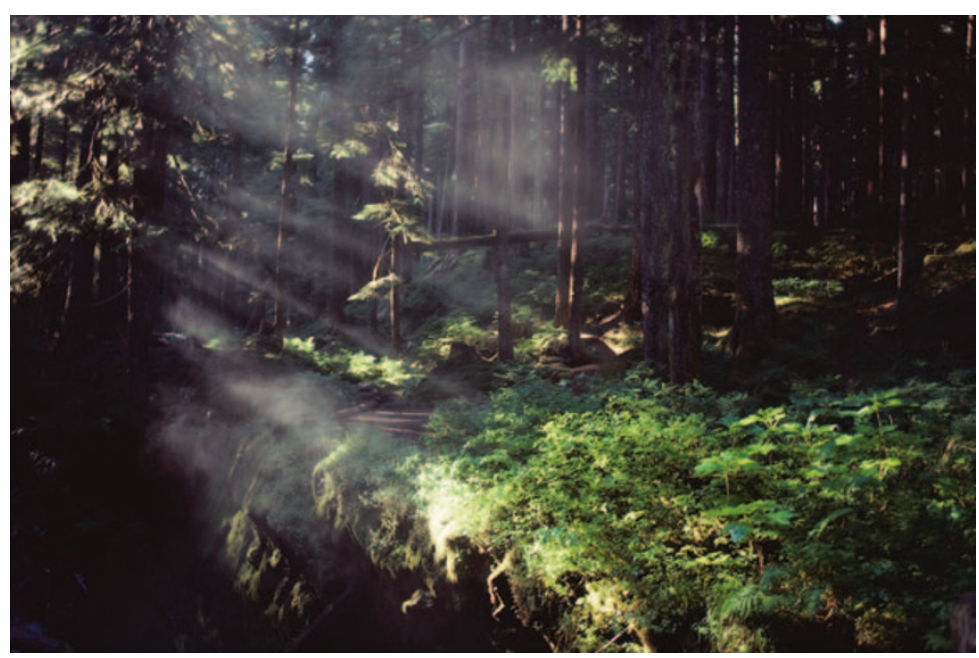

is composed of several highly selective TIR1-auxin interactions and a region that allows for more flexible binding. This partially promiscuous binding pocket allows for various auxin-like structures to be accommodated. Structures of TIR1-ASK1 with both auxin and an Aux/IAAderived peptide bound show that the Aux/IAA gene repressor binds on top of auxin, through extensive hydrophobic interactions with TIR1. This orients the repressor towards the predicted position of the SCF TIR1 subunit that has ubiquitin-conjugating activity.

Instead of an allosteric switch, the authors propose that auxin - sandwiched between TIR1 and the repressor - functions as 'molecular glue' that fills a cavity in the binding pocket and increases the surface area that is available for protein-protein interactions. The unusual mechanism by which auxin activates $S \mathrm{CF}^{\mathrm{TIR} 1}$ might have significance beyond plant biology: the principle that small molecules can influence proteinprotein interactions is an approach that might lead to new drugs for various diseases.

\section{James Pickett}

ORIGINAL RESEARCH PAPER Tan, X. et al.

Mechanism of auxin perception by the TIR1 ubiquitin ligase. Nature 446, 640-645 (2007) FURTHER READING Teale, W. D. et al. Auxin in action: signalling, transport and the control of plant growth and development. Nature Rev. Mol. Cell Biol. 7, 847-859 (2006)

WEB SITE

Ning Zheng's laboratory:

http://depts.washington.edu/zhenglab 